\title{
ANÁLISE DO COLAPSO SEGMENTAR DA CABEÇA FEMORAL NAS FRATURAS DO ACETÁBULO TRATADAS CIRURGICAMENTE
}

\author{
ANALYSIS OF THE SEGMENTAL IMPACTION OF FEMORAL HEAD \\ FOLLOWING AN ACETABULAR FRACTURE SURGICALLY MANAGED
}

Rodrigo Pereira Guimarães ${ }^{1}$, Camila Cohen Kaleka ${ }^{2}$, Carina Cohen², Daniel Daniachi ${ }^{3}$, Nelson Keiske Ono ${ }^{4}$, Emerson Kiyoshi Honda $^{5}$, Giancarlo Cavalli Polesello ${ }^{6}$, Walter Riccioli Junior ${ }^{7}$

\section{RESUMO}

Objetivo: Correlacionar a evolução radiográfica pós-operatória com as variáveis que acompanham as fraturas do acetábulo, a fim de definir o que tem valor preditivo no aparecimento do colapso segmentar da cabeça femoral. Métodos: Realizada análise retrospectiva de prontuários de pacientes submetidos à cirurgia de redução aberta e fixação interna do acetábulo. Em aproximadamente 35 anos, 596 pacientes foram tratados por fratura do acetábulo; 267 foram acompanhados pelo menos por dois anos. Os demais foram excluídos por não ter sido acompanhados pelo tempo mínimo, não haver dados suficientes no prontuário ou ter sido submetidos a tratamento não operatório. O acompanhamento dos pacientes foi feito por um de três cirurgiões do grupo, utilizando a escala clínica de Merle d’Aubigné e Postel e estudos radiológicos. Resultados: Somente duas variáveis estudadas, idade e qualidade da redução pós-operatória, apresentaram correlação estatisticamente significante com o colapso da cabeça femoral. Conclusões: A qualidade da redução, anatômica ou com desvio residual de até dois milímetros, apresenta evolução radiográfica satisfatória, diminuindo a probabilidade do colapso segmentar da cabeça femoral, achado que tem significância estatística.

Descritores - Acetábulo/lesões; Fraturas do quadril; Acetábulo/ cirurgia; Necrose da cabeça do fêmur

\begin{abstract}
Objective: Correlate the postoperative radiographic evaluation with variables accompanying acetabular fractures in order to determine the predictive factors for segmental impaction of femoral head. Methods: Retrospective analysis of medial files of patients submitted to open reduction surgery with internal acetabular fixation. Within approximately 35 years, 596 patients were treated for acetabular fractures; 267 were followed up for at least two years. The others were excluded either because their follow up was shorter than the minimum time, or as a result of the lack of sufficient data reported on files, or because they had been submitted to non-surgical treatment. The patients were followed up by one of three surgeons of the group using the Merle d'Aubigné and Postel clinical scales as well as radiological studies. Results: Only tow studied variables - age and amount of postoperative reduction- showed statistically significant correlation with femoral head impaction. Conclusions: The quality of reduction - anatomical or with up to $2 \mathrm{~mm}$ residual deviation - presents a good radiographic evolution, reducing the potential for segmental impaction of the femoral head, a statistically significant finding.
\end{abstract}

Keywords - Acetabulum/injuries; Hip fractures; Acetabulum / surgery; Femur head necrosis

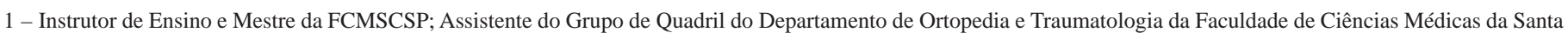
Casa de São Paulo (FCMSCSP), São Paulo, Brasil.

2 - Médica Residente do Departamento de Ortopedia e Traumatologia da FCMSCSP, São Paulo, Brasil.

3 - Médico Ortopedista; Voluntário do Grupo de Quadril do Departamento de Ortopedia e Traumatologia da FCMSCSP.

4 - Professor Assistente e Doutor da FCMSCSP; Chefe do Grupo de Quadril do Departamento de Ortopedia e Traumatologia da FCMSCSP, São Paulo, Brasil.

5 - Instrutor de Ensino e Doutor da FCMSCSP; Membro Sênior do Grupo de Quadril do Departamento de Ortopedia e Traumatologia da FCMSCSP, São Paulo, Brasil.

6 - Professor Assistente e Doutor da FCMSCSP; Assistente do Grupo de Quadril do Departamento de Ortopedia e Traumatologia da FCMSCSP, São Paulo, Brasil.

7 - Médico Ortopedista; Assistente do Grupo de Quadril do Departamento de Ortopedia e Traumatologia da FCMSCSP, São Paulo, Brasil.

Trabalho realizado no Departamento de Ortopedia e Traumatologia da Faculdade de Ciências Médicas da Santa Casa de São Paulo (FCMSCSP) -

Diretor: Prof. Dr. Osmar Avanzi.

Correspondência: Santa Casa de São Paulo - Departamento de Ortopedia e Traumatologia: Grupo do Quadril. Rua Cesário Motta Junior, 112. São Paulo, SP - Brasil.

E-mail: ddaniachi@hotmail.com 


\section{INTRODUÇÃO}

Com o aumento do número de traumatismos de alta energia, provocados principalmente por acidentes automobilísticos, aliado à melhoria nos sistemas de resgate dos pacientes politraumatizados, houve um incremento do número de pacientes com fraturas do acetábulo que chegam aos hospitais de referência ${ }^{(1)}$.

O acetábulo faz parte da articulação do quadril, cercada por ligamentos, músculos, vasos e nervos. Apresenta características anatômicas individuais, o que torna difícil o acesso para os cirurgiões ortopedistas menos experientes. Porém, a partir de 1964 com os estudos de Judet et al, que correlacionaram as características anatômicas com os aspectos radiográficos e os achados intraoperatórios, elaborando uma classificação topográfica capaz de orientar na via de acesso, o entendimento dessas lesões tornou-se mais lógico ${ }^{(2,3)}$.

Atualmente, o tratamento cirúrgico das fraturas desviadas do acetábulo é um consenso. Porém, o grande número de complicações inerentes a essas fraturas e que acompanham o ato cirúrgico pode influenciar no resultado final ${ }^{(4-10)}$.

Uma das complicações que aparece precocemente é o colapso segmentar da cabeça femoral, citado por alguns autores como desgaste articular e por outros como necrose ${ }^{(2,4,7,10-12)}$.

O objetivo deste estudo é correlacionar a evolução radiográfica pós-operatória com as variáveis que acompanham as fraturas do acetábulo a fim de definir o que tem valor preditivo no aparecimento do colapso segmentar da cabeça femoral.

\section{MÉTODOS}

Realizada avaliação retrospectiva de 596 prontuários de pacientes submetidos ao tratamento de fraturas acetabulares no Departamento de Ortopedia e Traumatologia da Santa Casa de Misericórdia de São Paulo, Pavilhão "Fernandinho Simonsen”, no período compreendido entre 1972 e 2006. Desses, 267 prontuários foram submetidos à análise. Excluímos os pacientes submetidos ao tratamento não operatório, os que não tinham tempo de pós-operatório mínimo de dois anos e quando não traziam as informações completas necessárias ao nosso estudo.

Cada paciente era portador de uma única fratura; o lado esquerdo foi atingido em 152 casos (56,9\%) e o lado direito em 115 casos (43,1\%). O intervalo entre a fratura e a fixação variou entre um e 90 dias, sendo a média de 13,4 dias. O tempo de seguimento foi de 24 meses e, ao final, foi realizada avaliação. A idade dos pacientes variou de 11 a 87 anos, sendo a média de 33,9 anos por ocasião da fratura. Quanto ao sexo, foram tratados 72 (27,0\%) mulheres e 195 (73,0\%) homens.

Todos os pacientes foram submetidos à avaliação radiográfica, exame padronizado no serviço, com a ampola a um metro do chassi da película. Avaliadas radiografias em três incidências: anteroposterior, alar e foraminal. $\mathrm{O}$ desvio inicial foi medido nas três incidências e o maior valor detectado foi anotado (Figura 1). A avaliação radiográfica pós-operatória foi realizada do mesmo modo e o maior desvio detectado foi registrado (Figura 2).

O colapso da cabeça femoral foi caracterizado pela perda da esfericidade da cabeça femoral, com achatamento da área de carga, geralmente associada com esclerose da área subcondral (Figura 3).
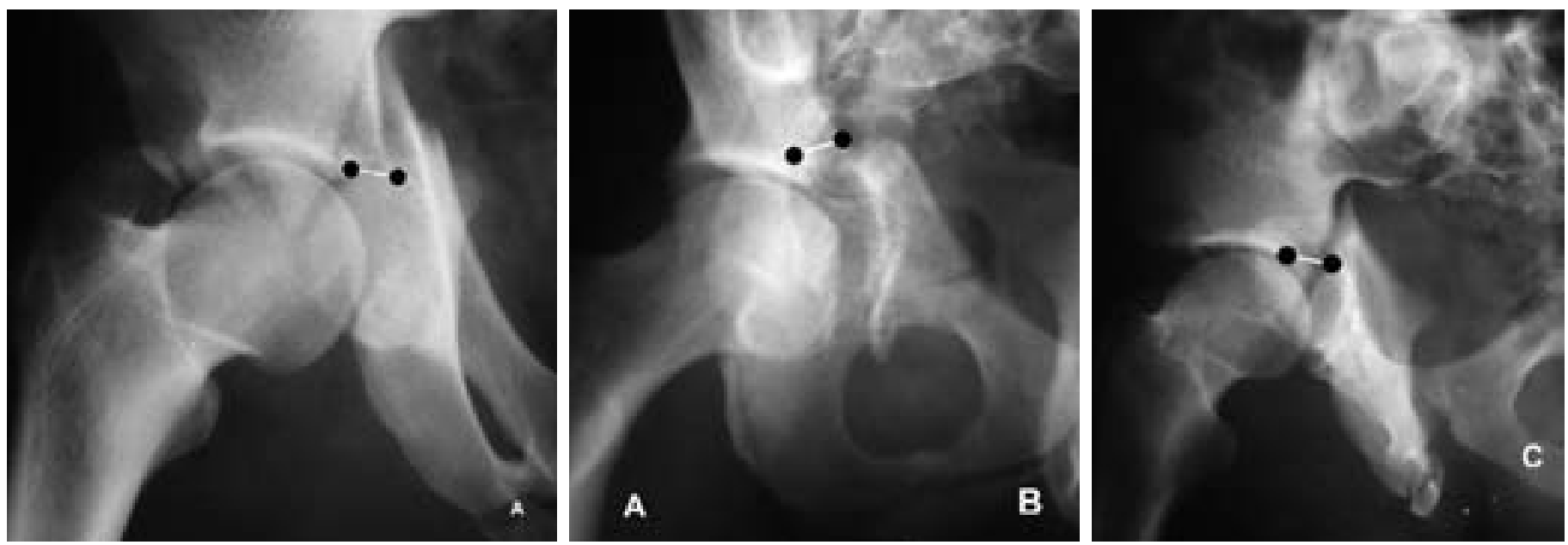

Figura 1 - Medição do desvio inicial: incidência anteroposterior (A), foraminal (B) e alar (C) 


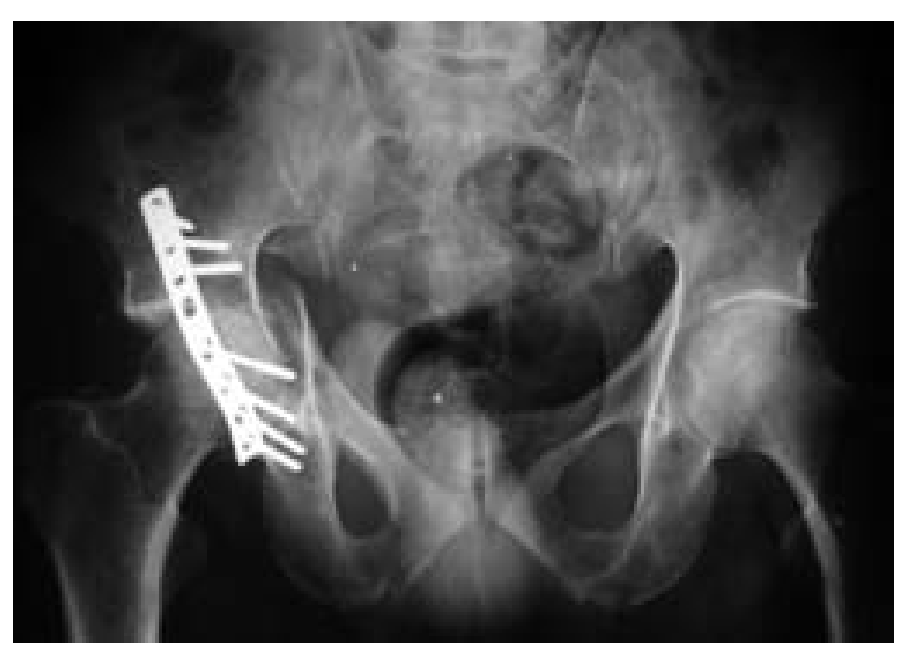

Figura 2 - Redução inicial da fratura com manutenção do desvio

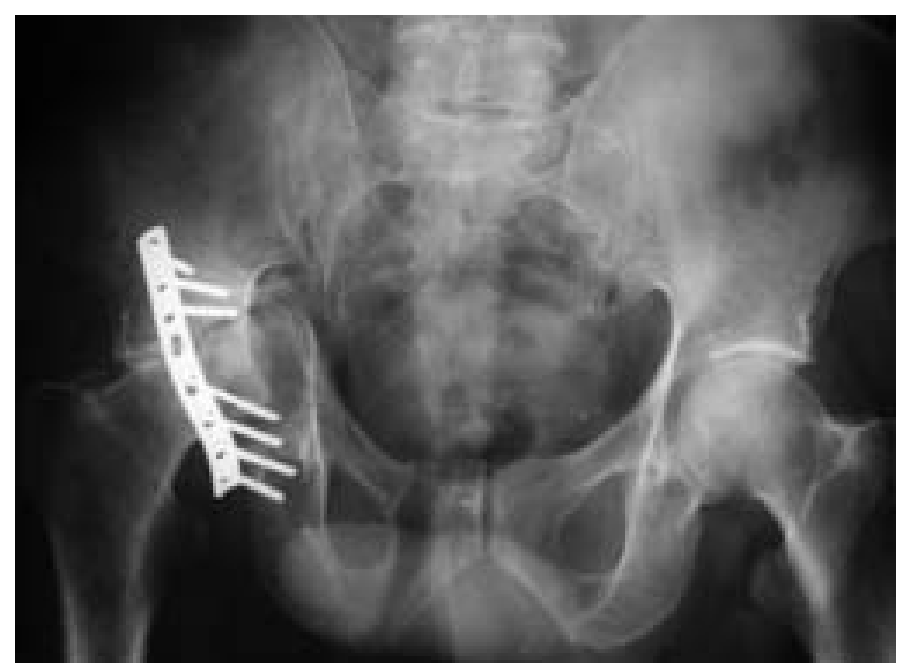

Figura 3 - Colapso segmentar da cabeça femoral

Com relação ao tipo de trauma, 133 (49,8\%) decorreram de acidente de automóvel, 50 (18,7\%) de atropelamento, 46 (17,2\%) de queda de altura e 38 (14,2\%) de acidente motociclístico.

Quanto ao desvio inicial, pode-se observar que em 24 fraturas (9\%) era menor que $4 \mathrm{~mm}$, em 94 fraturas $(35,2 \%)$ apresentava-se entre 4 e $10 \mathrm{~mm}$ e em 149 fraturas (55,8\%) era maior que $10 \mathrm{~mm}$.

Observou-se luxação em 91 casos (34,1\%), cominuição em 95 casos (35,6\%), fratura da pelve em 55 casos (20,6\%), lesão do nervo ciático em 29 casos (10,9\%) e lesão na cabeça femoral em 13 casos (4,9\%).

O tratamento operatório foi indicado quando havia desvio inicial maior que $2 \mathrm{~mm}$ ou em fraturas instáveis. Algumas fraturas com indicação cirúrgica, no entanto, foram tratadas incruentamente devido a problemas clínicos associados ao trauma.
Com relação ao desvio residual pós-operatório, observou-se redução anatômica em 151 casos (56,5\%), com desvio até $2 \mathrm{~mm}$ em 60 casos (22,4\%); a redução foi maior que $2 \mathrm{~mm}$ em 56 casos (20,9\%).

O acompanhamento ambulatorial foi realizado por um de três cirurgiões do grupo, que lançaram mão da escala de avaliação clínica de Merle d’Aubigné e Postel $^{(13)}$ e estudos radiológicos com retorno no primeiro mês e terceiro mês; a partir de então, foram marcados retornos a cada três meses até que se completasse um ano de pós-operatório. No segundo ano os retornos eram semestrais; a partir de então, os passaram a ser anuais.

As variáveis foram resumidas e representadas pelas estatísticas descritivas pertinentes: média, desvio padrão (dp), mediana, valores mínimos e máximos ou frequência absoluta e relativa (\%).

Foram aplicados o teste $t$ de Student ou o teste de Mann-Whitney para amostras não relacionadas na comparação entre os grupos sem e com presença de colapso quanto às variáveis quantitativas, dependendo se as variáveis dentro dos grupos seguem distribuição normal ou não.

Os grupos foram comparados quanto às variáveis qualitativas pelo teste do qui-quadrado de Pearson ou pelo teste do qui-quadrado da razão de verossimilhança.

Foi adotado o nível de significância de 0,05 ou 5\% (alfa $\leq 0,05 ; \mathrm{p} \leq 0,05)$ e utilizado o programa estatístico SPSS versão 15.0 para Windows para todas as análises.

\section{RESULTADOS}

Foram analisados 267 prontuários completos de pacientes com fraturas acetabulares, tratados cirurgicamente, dos quais 16 (6,0\%) evoluíram para colapso da cabeça femoral.

Correlacionou-se a incidência de colapso com sexo, idade, tipo de trauma, classificação de Judet et $a^{(2)}$, desvio, luxação, cominuição, fratura da cabeça do fêmur, fratura da pelve, lesão do nervo ciático, tipo de tratamento, via de acesso e qualidade da redução pósoperatória.

Somente duas variáveis analisadas, idade e redução pós-operatória, apresentaram correlação estatisticamente significante com o colapso da cabeça femoral. A qualidade da redução é fator preditivo quanto ao aparecimento do colapso segmentar da cabeça femoral (Tabela 1). 
Tabela 1 - Variáveis estatisticamente significantes com o colapso da cabeça femoral (idade e redução pós-operatória)

\begin{tabular}{|c|c|c|c|c|}
\hline \multirow{2}{*}{ Variáveis } & \multicolumn{2}{|c|}{ Colapso } & \multirow{2}{*}{$\begin{array}{c}\text { Total } \\
(n=267)\end{array}$} & \multirow{2}{*}{$p$} \\
\hline & Não $(n=251)$ & $\operatorname{Sim}(n=16)$ & & \\
\hline \multicolumn{5}{|l|}{ Idade (anos) } \\
\hline Média (dp) & $33,6(12,8)$ & $40,7(15,4)$ & $34,0(13,0)$ & \multirow{3}{*}{0,034 * } \\
\hline Mediana & 30 & 37 & 31 & \\
\hline Mínimo - máximo & $14-87$ & $11-65$ & $11-87$ & \\
\hline \multicolumn{5}{|l|}{$\begin{array}{l}\text { Redução pós- } \\
\text { operatória n (\%) }\end{array}$} \\
\hline 0 & $149(59,4)$ & $2(12,5)$ & $151(56,6)$ & \multirow{3}{*}{$<0,001$} \\
\hline 2 & $56(22,3)$ & $4(25,0)$ & $60(22,5)$ & \\
\hline$>2$ & $46(18,3)$ & $10(62,5)$ & $56(21,0)$ & \\
\hline
\end{tabular}

\section{DISCUSSÃO}

As fraturas do acetábulo são classicamente causadas por traumas de alta energia. Por ser fraturas resultantes desses traumas, atingem com maior frequência indivíduos jovens na fase produtiva, uma vez que estão mais expostos a esse tipo de ocorrência ${ }^{(1,4,14)}$.

As formas de tratamento variaram com o decorrer dos anos. Até a década de 70, o tratamento incruento foi o mais utilizado, porém, os maus resultados e o fato de esse tipo de fratura incidir numa população jovem e economicamente ativa estimularam os ortopedistas a buscarem novas formas de tratamento ${ }^{(4,6,10)}$.

Judet et $a l^{(2)}$ promoveram uma mudança significativa na forma de abordagem dessas fraturas, pois com seus estudos permitiram a sistematização do tratamento. A partir de então, o tratamento operatório, que era somente uma opção, passou a ser a escolha ${ }^{(4)}$.

Em decorrência das causas dessas fraturas, já era de esperar que viessem acompanhadas de complicações. A complicação primária nas fraturas do acetábulo é a artrose pós-traumática; entre outras complicações podemos citar: pseudoartrose, encurtamento do membro afetado, miosite ossificante, alterações neurológicas e vasculares, colapso segmentar, necrose da cabeça femoral e outras ${ }^{(2,4,5,15-21)}$.

Entre as complicações apontadas com maior frequência estão a osteoartrose do quadril e a necrose avascular da cabeça femoral, ambos os diagnósticos feitos radio graficamente $e^{(4,10,12,17,22,23)}$. Porém, Catto já adverte que a necrose avascular é uma alteração tecidual que não se manifesta radiograficamente e que o termo necrose provavelmente é o reconhecimento tardio do colapso da cabeça femoral ${ }^{(24)}$.
Gruen et $a l^{(25)}$ relatam que a necrose avascular da cabeça femoral secundária ao trauma é citada historicamente como complicação da fratura acetabular, porém, afirmam que a impactação é a causa das alterações na forma da cabeça e não a necrose avascular. Relatam que os chamados "sinais radiográficos de necrose avascular da cabeça femoral” não apresentam correlação clínica. Referem que, se a osteoartrose pós-traumática se desenvolver na presença de cartilagem acetabular viável, é primariamente resultado da distribuição alterada das forças de pressão.

A diminuição da área de contato da superfície articular da cabeça femoral em relação ao acetábulo, causada pela má redução da fratura, acarreta o aumento da pressão localizada na cartilagem e no osso subjacente. Isso resulta na perda do espaço articular e, algumas vezes, no desgaste da cabeça femoral ${ }^{(25,26)}$.

Na opinião dos autores, os princípios gerais do tratamento das fraturas articulares devem ser aplicados à articulação do quadril, de maneira mais acentuada, por se tratar de uma articulação de carga com grande amplitude de movimento.

O colapso da cabeça femoral é costumeiramente atribuído à necrose avascular, transferindo a responsabilidade do ocorrido para o trauma inicial e para a lesão avascular associada.

$\mathrm{Na}$ análise dos casos houve correlação estatisticamente significante entre o colapso e a má redução da fratura e, portanto, acreditamos que essa seja uma variável determinante do processo. Não queremos excluir a necrose avascular como um dos fatores que também venham causar o colapso da cabeça femoral.

A média de idade do grupo com colapso foi estatisticamente significante maior do que a do grupo sem colapso, com 40,7 anos e 33,6 anos, respectivamente, em nossa casuística. Tais dados devem ser observados com cautela devido ao pequeno número de casos com colapso.

\section{CONCLUSÕES}

A análise dos prontuários de 267 pacientes (267 quadris), tratados com fraturas acetabulares e a revisão da literatura pertinente nos permitem concluir que a qualidade da redução, anatômica ou com desvio residual de até $2 \mathrm{~mm}$, apresenta evolução radiográfica satisfatória, diminuindo a probabilidade do colapso segmentar da cabeça femoral, achado que tem significância estatística. 


\section{REFERÊNCIAS}

1. Geoghegan JM, Longdon EJ, Hassan K, Calthorpe D. Acetabular fractures in the UK. What are the numbers? Injury. 2007;38(3):329-33.

2. Judet R, Judet J, Letournel E. Fractures of the acetabulum: classification and surgical approaches for open reduction. Preliminary Report. J Bone Joint Surg Am. 1964;46:1615-46.

3. Beaulé PE, Dorey FJ, Matta JM. Letournel classification for acetabular fractures. Assesment of interobserver and intraobserver reliability. J Bone Joint Surg Am. 2003;85(9):1704-9.

4. Giannoudis PV, Grotz MR, Papakostidis C, Dinopoulos H. Operative treatment of displaced fractures of the acetabulum: a meta-analysis. J Bone Joint Surg Br. 2005;87(1):2-9.

5. Aristide RSA, Honda E, Polesello G, Fernandez MS. Fratura em "T" do acetábulo: análise de 45 casos. Rev Bras Ortop. 1996;31(11):919-24.

6. Knop T, Silva LHP, Laghi R. Fraturas acetabulares: resultados de tratamento cirúrgico. Rev Bras Ortop. 1996;31(10):825-30.

7. Matta JM. Fractures of the acetabulum: accuracy of reduction and clinical results in patients managed operatively within three weeks after the injury. J Bone Joint Surg. 1996;78(11):1632-45.

8. Liebergall M, Mosheiff R, Low J, Goldvirt M, Matan Y, Segal D. Acetabular fractures. Clinical outcome of surgical treatment. Clin Orthop Relat Res. 1999;(366):205-16.

9. Barbosa ALH, Schutz PC, Pavan L. Tratamento cirúrgico das fraturas de acetábulo: estudo retrospectivo de 48 casos. Acta Ortop Bras. 2000;8(3):140-3.

10. Laird A, Keating JF. Acetabular fractures: a 16-year prospective epidemiological study. J Bone Joint Surg Br. 2005;87(7):969-73.

11. Letournel E. Acetabulum fractures: classification and management. Clin Orthop Relat Res. 1980;(151):81-106.

12. Köberle G, Miranda JB, Yamanaka E, Grava ALS, Belangero WD. Fraturas do acetábulo: estudo dos resultados clínicos do tratamento cirúrgico precoce e tardio. Rev Bras Ortop. 1993;28(6):361-6.

13. D'Aubgné RM, Postel M. Functional results of hip arthroplasty with acrylic prosthesis. J Bone Joint Surg Am. 1954;36(3):451-75.
14. Glas PY, Fessy MH, Béjui-Hugues J. Surgical treatment of acetabular fractures: outcome in a series of 60 consecutive cases. Rev Chir Orthop Reparatrice Appar Mot. 2001;87(6):529-38.

15. Vaughan GT. Central dislocation of the femur. Surg Gynec Obstet. 1912;15:249-51.

16. Armstrong JR. Traumatic dislocation of the hip joint. Review of one hundred and one dislocations. J Bone Joint Surg Am. 1948;30B(3):430-45

17. Letournel E. Fractures of the acetabulum. A study of a serie of 75 cases. J Chronic Dis. 1961;82:47-87.

18. Mayo KA. Open reduction and internal fixation of fractures of the acetabulum. Clin Orthop Relat Res. 1994;(305):31-7.

19. Mayo KA, Letournel E, Matta JM, Mast JW, Johnson EE, Martimbeau CL. Surgical revision of malreduced acetabular fractures. Clin Orthop Relat Res. 1994;(305):47-52.

20. Issack PS, Toro JB, Buly RL, Helfet DL. Sciatic nerve release following fracture or reconstructive surgery of the acetabulum. J Bone Joint Surg Am. 2007;89(7):1432-7.

21. Madhu R, Kotnis R, Al-Mousawi A, Barlow N, Deo S, Worlock P, et al. Outcome of surgery for reconstruction of fracture of the acetabulum: the time dependent effect of delay. J Bone Joint Surg Br. 2006;88(9):1197-203.

22. Rowe CR, Lowell D. Prognosis of fractures of the acetabulum. J Bone Joint Surg Am. 1961;43(1):30-58.

23. Ruesch PD, Holdener $\mathrm{H}$, Ciaramitaro M, Mast JW. A prospective study of surgically treated acetabular fractures. Clin Orthop Relat Res. 1994;(305):38-46.

24. Catto M. A histological study of avascular necrosis of the femoral head transcervical fracture. J Bone Joint Surg Br. 1965;47(4):749-75.

25. Gruen GS, Mears DC, Tauxe WN. Distinguishing avascular necrosis from segmental impaction of the femoral head following an acetabular fracture: preliminary report. J Orthop Trauma. 1988;2(1):5-9.

26. Olson SA, Bay BK, Chapman MW, Sharkey NA. Biomechanical consequences of posterior wall acetabular fractures and repair. J Bone Joint Surg Am. 1995;77(8):1184-92. 